Are regi onal vari at i ons i $n$ act i vity of di spat cher - assi st ed car di opul monary

resusci tat i on associ at ed wi th out- of - hospi tal car di ac ar rest s out comes? A nat i on- wi de popul at i on- based cohort st udy

\begin{tabular}{|l|l|}
\hline 著者 & 西 大樹 \\
\hline 著者別表示 & $\mathrm{N}$ shi Tai ki \\
\hline $\begin{array}{l}\mathrm{j} \text { our nal or } \\
\text { publ i cat i on } \mathrm{ti} \text { t l e }\end{array}$ & 博士論文本文Ful I \\
\hline 学位授与番号 & 13301甲第4356号 \\
\hline 学位名 & 博士 (医学) \\
\hline 学位授与年月日 & 2016- 03 22 \\
\hline URL & ht t p: //hdl . handl e. net /2297/45952 \\
\hline
\end{tabular}


Clinical paper

\title{
Are regional variations in activity of dispatcher-assisted cardiopulmonary resuscitation associated with out-of-hospital cardiac arrests outcomes? A nation-wide population-based cohort study
}

\author{
Taiki Nishi ${ }^{a}$, Takahisa Kamikura ${ }^{a}$, Akira Funada ${ }^{\mathrm{b}}$, Yasuhiro Myojo ${ }^{\mathrm{c}}$, Tetsuya Ishida ${ }^{\mathrm{d}}$, \\ Hideo Inaba ${ }^{\mathrm{a}, *}$ \\ a Department of Emergency Medical Science, Kanazawa University Graduate School of Medicine, 13-1 Takara-machi, Kanazawa, Ishikawa 920-8641, Japan \\ ${ }^{\mathrm{b}}$ Emergency Medical Centre, Kanazawa University Hospital, 13-1 Takara-machi, Kanazawa, Ishikawa 920-8641, Japan \\ ' Emergency Medical Centre, Ishikawa Prefectural Central Hospital, 2-1 Kuratsuki-Higashi, Kanazawa, Ishikawa 920-8201, Japan \\ d Emergency Department, Kaga Citizen's Hospital, 65 Hachikenmichi, Daishoji, Kaga 922-0057, Japan
}

\section{A R T I C L E I N F O}

\section{Article history:}

Received 10 March 2015

Received in revised form 6 August 2015

Accepted 11 October 2015

\section{Keywords:}

Cardiopulmonary resuscitation

Dispatcher-assisted

Cross-regional comparison

Out-of-hospital cardiac arrest

Survival

Pre-arrival instructions

\begin{abstract}
A B S T R A C T
Aim: Dispatcher-assisted cardiopulmonary resuscitation (DA-CPR) impacts the rates of bystander CPR (BCPR) and survival after out-of-hospital cardiac arrests (OHCAs). This study aimed to elucidate whether regional variations in indexes for BCPR and emergency medical service (EMS) may be associated with OHCA outcomes.

Methods: We conducted a population-based observational study involving 157,093 bystander-witnessed, resuscitation-attempted OHCAs without physician involvement between 2007 and 2011. For each index of BCPR and EMS, we classified the 47 prefectures into the following three groups: advanced, intermediate, and developing regions. Nominal logit analysis followed by multivariable logistic regression including OHCA backgrounds was employed to examine the association between neurologically favourable 1-month survival, and regional classifications based on BCPR- and EMS-related indexes.

Results: Logit analysis including all regional classifications revealed that the number of BLS training course participants per population or bystander's own performance of BCPR without DA-CPR was not associated with the survival. Multivariable logistic regression including the OHCA backgrounds known to be associated with survival (BCPR provision, arrest aetiology, initial rhythm, patient age, time intervals of witness-to-call and call-to-arrival at patient), the following regional classifications based on DA-CPR but not on EMS were associated with survival: sensitivity of DA-CPR [adjusted odds ratio (95\% confidence intervals) for advanced region; those for intermediate region, with developing region as reference, 1.277 (1.131-1.441); $1.162(1.058-1.277)]$; the proportion of bystanders to follow DA-CPR [1.749 (1.554-1.967); $1.280(1.188-1.380)]$.

Conclusions: Good outcomes of bystander-witnessed OHCAs correlate with regions having higher sensitivity of DA-CPR and larger proportion of bystanders to follow DA-CPR.
\end{abstract}

(C) 2015 Elsevier Ireland Ltd. All rights reserved.

\section{Introduction}

Regional variations in the rate of survival after out-of-hospital cardiac arrests (OHCAs) have been reported. ${ }^{1-4}$ These variations

\footnotetext{
* Corresponding author.

E-mail addresses: ntaiki24@yahoo.co.jp (T. Nishi), kamitaka911@gmail.com (T. Kamikura), funada@med.kanazawa-u.ac.jp (A. Funada), yasuhiromyojo@yahoo.co.jp (Y. Myojo),m.kawamoto@city.kaga.lg.jp (T. Ishida), hidinaba@med.kanazawa-u.ac.jp (H. Inaba).
}

are likely due to uneven distribution of high-grade management of OHCAs in both prehospital and in-hospital settings. ${ }^{4}$

Rapid initiation of cardiopulmonary resuscitation (CPR) is an important determinant of survival after OHCA. ${ }^{5,6}$ Nevertheless, laypersons who witness OHCAs rarely start bystander CPR (BCPR) on their own initiative. ${ }^{2,3}$ Dispatcher-assisted CPR (DA-CPR) increases the rate of $B C P R$ performance and may improve survival after OHCAs. ${ }^{7,8}$ The impact of DA-CPR on survival may depend on the dispatcher's experience ${ }^{8}$ and quality of the DA-CPR. ${ }^{9,10}$

Therefore, regional variations in the provision of high-quality DA-CPR may be one of the causes for variations in the rates 
of BCPR and OHCA outcomes. The quality of DA-CPR should be measured in terms of early and proper recognition of savable cardiac arrests. ${ }^{7,8,10}$ This study was conducted to elucidate whether regional variations in indexes related to DA-CPR and BCPR may be associated with better outcomes of OHCAs.

\section{Methods}

\section{Data collection}

We obtained permission from the Fire and Disaster Management Agency (FDMA) of Japan to analyse the FDMA data prospectively collected for 388,221 OHCAs that occurred from January 2007 to December 2011. The FDMA database was created by a prospective, nationwide, population-based registry system of OHCAs according to the Utstein style. ${ }^{11}$ This study was designed by a group comprising members of the Ishikawa Medical Control Council and their collaborators, and it was approved by the review board of Ishikawa Medical Control Council.

FDMA databases include the following information recommended at the Utstein International Conference ${ }^{11}$ : patients backgrounds, arrest witness, aetiology of OHCA (presumed cardiac or non-cardiac), derivation of BCPR (with or without DA-CPR instruction), type of BCPR (conventional or compression-only), initial cardiac rhythm, estimated time of collapse, times of CPR initiation by bystander and emergency medical technician (EMT), and times of EMT arrival on the OCHA site and in the hospital, onemonth (1-M) survival, and neurologically favourable 1-M survival (cerebral performance category, 1 or 2). The times of collapse and BCPR initiation and type of BCPR were estimated from the EMT's interview of the bystander. Cardiac or non-cardiac origin was clinically determined by EMTs in collaboration with physicians. The fire departments obtained information on 1-M survivals and neurological outcome from the hospital records. The FDMA logically checked data integrated in the registry system and, if necessary, requested the respective fire department to correct and complete the data.

Population, geography, emergency medical service (EMS) and basic life support training

Japan is composed of 47 prefectures. Population and geographic data in each prefecture were collected from the 2007-2012 Japan Census data. ${ }^{12}$

In 2011, 791 fire departments had 4,965 ambulance teams. All fire departments principally have a one-tiered dispatch system. Fire departments play a major role in educating citizens regarding basic life support (BLS). Numbers of ambulances, ambulance dispatches, and participants in BLS training courses held by fire departments in each prefecture were collected from a nation-wide database created by the FDMA. ${ }^{13}$ In Japan, new driver license applicants are obligated to receive BLS training in qualified driving schools. ${ }^{14}$ The number of new driver license applicants in each prefecture was collected from the Statics in Driver License in Japan. ${ }^{15}$ The total number of participants in BLS training courses conducted by fire departments and authorized driving schools was calculated in each prefecture.

Unless an OHCA patient is obviously dead or presents postmortem changes, EMTs must not terminate resuscitation at the scene. In addition to basic life support, EMTs may use airway adjuncts, including suprapharyngeal and laryngeal masks, and may commence a peripheral venous infusion of Ringer's lactate. However, only authorized and specially trained EMTs are permitted to insert tracheal tubes and administer intravenous epinephrine.

At the end of 2006, the Japan Resuscitation Council (JRC) announced similar guidelines to those of the AHA (JRC Guidelines
2005). ${ }^{16}$ Therefore, BLS was substantially performed in accordance with JRC Guidelines 2005 in the period of 2007-2011. Provision of DA-CPR was encouraged by FDMA in 1999. However, a standard protocol based on the JRC guidelines for DA-CPR and education of dispatcher was released in 2013 (after the study period of this study). Thus, basically each fire department provided DA-CPR instruction on callers according to different protocols during the study period.

\section{Data selection}

From the 388,221 OHCAs that occurred from January 2007 to December 2011, we first extracted a dataset comprising 192,794 bystander-witnessed OHCA without any prehospital involvement of physicians, eliminating cases with physician involvement as per the following reasons: (1) some of these cases received prehospital advanced life support (ALS) performed by physicians on duty ${ }^{17} ;(2)$ most of these OHCAs occurred in medical offices and sanatoriums or during home visit by physicians, and physicians on duty played primary roles in the treatment and transportation of patients; and (3) these physicians should not be categorized as bystanders according to the Utstein recommendations. ${ }^{11}$ Then, we excluded the following cases lacking the essential information for the analysis: 4,539 cases lacking fundamental time records, 12 cases with unknown patient backgrounds, and 2,765 cases with unknown DA-CPR or type of BCPR. Finally, we selected 157,093 bystander-witnessed cases with a complete dataset available (Fig. 1).

This study analysed only the bystander-witnessed cases. As stated in the Utstein Recommendation, ${ }^{11}$ this implies that the bystander was present when the patient went into cardiac arrests before EMT arrival at patients.

\section{Calculation of three indexes related to $D A-C P R$ and $B C P R$}

According to the existence and timing of BCPR and DA-CPR, BCPR was divided into four groups: BCPR following DA-CPR, BCPR on bystander's own initiative (without DA-CPR), no BCPR despite DA-CPR, and no BCPR without DA-CPR. We calculated the following three indexes associated with DA-CPR and BCPR: sensitivity of DA-CPR (= the number of cases for which DA-CPR was attempted divided by the number of cases that did not receive BCPR on bystander's own initiative); proportion of bystanders to follow DACPR (= the number of cases that received BCPR following DA-CPR divided by the number of cases for which DA-CPR was attempted); bystander's own performance of BCPR (= the number of cases that received $B C P R$ on bystander's own initiative divided by the number of cases for which DA-CPR was not attempted). These definitions and calculations from the four groups of BCPR are summarized in Table 1 . We determined these three indexes and the rate of BCPR in each prefecture.

\section{Calculations of EMS-related indexes}

From the data on population, geography, EMS system, and driver license applicants, ${ }^{12,13,15}$ we calculated the following indexes that may be related to the incidences of BCPR: annual number of BLS training course participants per population and proportion of aged ( $\geqq 65$ years) population. ${ }^{14}$ Furthermore, we calculated the following indexes that may be related to EMS response, the transportation of patients and ACLS procedures by paramedics: numbers of emergency hospitals and ambulance teams per $100 \mathrm{~km}^{2}$ of residential area, annual number of emergency medical dispatches per one ambulance team, and the rates of tracheal intubation and adrenalin administration by paramedics. 


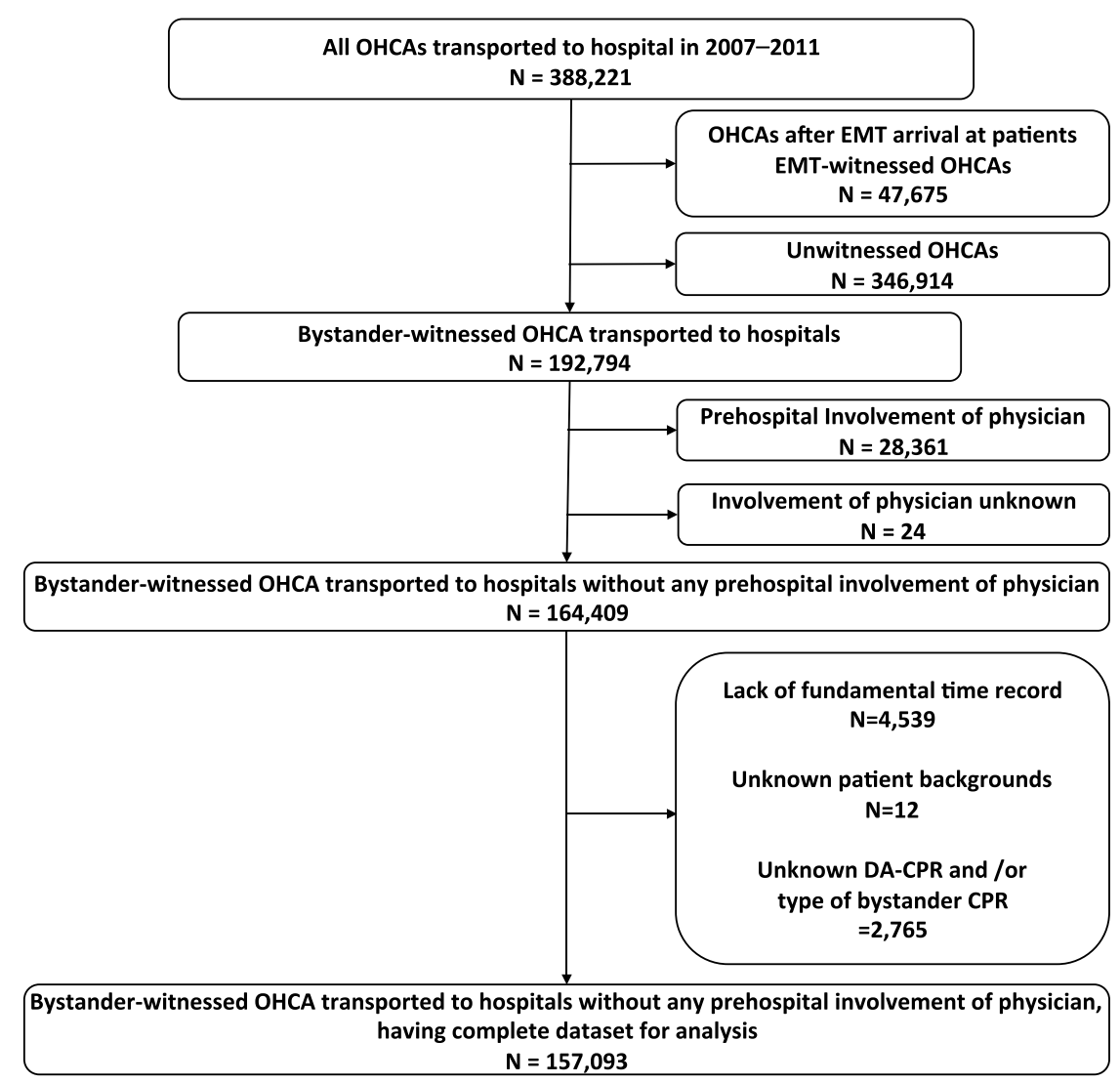

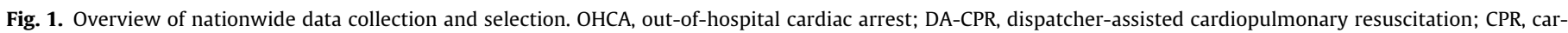
diopulmonary resuscitation.

Table 1

Definitions of three indexes related to DA-CPR and BCPR.

\begin{tabular}{|c|c|}
\hline Index & Definitions \\
\hline Sensitivity of DA-CPR & $\begin{array}{l}\text { [Number of bystander-witnessed OHCA cases } \\
\text { for which DA-CPR was attempted]/[Number of } \\
\text { bystander-witnessed OHCA cases that did not } \\
\text { receive } B C P R \text { on bystander's own initiative] } \\
=([\text { Number of cases in the BCPR following } \\
D A-C P R \text { group] + [Number of cases in the no } \\
B C P R \text { despite DA-CPR group] }) /([\text { Number of all } \\
\text { bystander-witnessed cases included in the } \\
\text { study] - [Number of cases in the BCPR on } \\
\text { bystander's own initiative group]) }\end{array}$ \\
\hline $\begin{array}{l}\text { Proportion of } \\
\text { bystanders to follow } \\
\text { DA-CPR (the rate of } \\
\text { DA-CPR accepted by } \\
\text { bystanders) }\end{array}$ & $\begin{array}{l}\text { [Number of bystander-witnessed OHCA cases } \\
\text { that received BCPR following } \\
\text { DA-CPR]/[Number of bystander-witnessed } \\
\text { OHCA cases for which DA-CPR was attempted] } \\
=[\text { Number of cases in the } B C P R \text { following } \\
D A-C P R \text { group]/([Number of cases in the } B C P R \\
\text { following } D A-C P R \text { group] + (Number of cases in } \\
\text { the no } B C P R \text { despite } D A-C P R \text { group]) }\end{array}$ \\
\hline $\begin{array}{l}\text { Bystander's own } \\
\text { performance of BCPR }\end{array}$ & $\begin{array}{l}\text { [Number of bystander-witnessed OHCA cases } \\
\text { that received BCPR on bystander's own } \\
\text { initiative]/[Number of bystander-witnessed } \\
\text { OHCA cases for which DA-CPR was not } \\
\text { attempted] } \\
\text { = [Number of cases in the BCPR on bystander's } \\
\text { own initiative group]/([Number of cases in the } \\
\text { BCPR on bystander's own initiative } \\
\text { group] + [Number of cases in the no BCPR } \\
\text { without DA-CPR group]) }\end{array}$ \\
\hline
\end{tabular}

$\overline{\mathrm{CPR} \text {, cardiopulmonary resuscitation; DA-CPR, dispatcher-assisted cardiopulmonary }}$ resuscitation; BCPR, bystander cardiopulmonary resuscitation. BCPR on bystander's own initiative = bystander-initiated CPR without DA-CPR.
Study endpoints and outcomes

The primary endpoint of this study was neurologically favourable 1-M survival (cerebral performance category 1 or 2 ). ${ }^{18}$

\section{Statistical analysis}

Data were analysed using JMP 11 Pro (SAS Institute, Cary, NC, USA) and/or a computer software by Preacher. ${ }^{19}$

Correlations among prefectural rates of neurologically favourable 1-M survival and calculated indexes were screened by correlation analysis with adjustment of number of OHCAs in each prefecture. Then, we classified the 47 prefectures into the following three groups in each index using entropy splitting with the rate of neurologically favourable 1-M survival as response variable: advanced, intermediate, and developing regions.

Differences across groups for nominal variables were assessed using the $\chi^{2}$ test with Yates' correction followed by calculation of unadjusted odds ratios (ORs) and $95 \%$ confidence intervals (CI). The Wilcoxon rank sum test was applied for nonparametric comparisons of continuous variables.

Multiple logistic regression analysis was used to examine the association between neurologically favourable 1-M survival, OHCA backgrounds, and regional classification based on BCPR- and EMSrelated indexes. We sequentially introduced groups of variables into the model: first, basic variables known to be associated with OHCA outcomes, then variables identified as significant in the univariable analysis in a stepwise manner to obtain the lowest Bayesian information criterion (BIC). The generalized $R^{2}$ of the final model was computed to measure the fit of the regression model. 
For each analysis, the null hypothesis was evaluated at a two-sided significance level of $p<0.05$, with $95 \% \mathrm{CI}$ calculated using profile likelihood.

\section{Results}

Variations of BCPR-related indexes and classification of prefectures

The number of OHCAs, showed by the size of symbols in Fig. 2, widely differed in the 47 prefectures because of differences in population. The sensitivity of DA-CPR, proportion of bystanders to follow DA-CPR, and bystander's own performance of BCPR in the 47 prefectures was $32.4-73.6 \%, 47.7-78.4 \%$, and $24.2-41.4 \%$, respectively. Correlation analysis revealed the following findings: (1) the sensitivity of DA-CPR and proportion of bystanders to follow DA-CPR positively correlated to the rate of neurologically favourable 1-M survival (correlation coefficient; 95\% CI, 0.306; 0.302-0.311 and 0.652; 0.649-0.655, respectively), (2) bystander's own performance of BCPR negatively correlated to the survival rate $(-0.294 ;-0.299$ to -0.290$)$, and (3) the three indexes associated with BCPR correlated to each other.

\section{Variations of EMS-related indexes and classification}

Annual number of BLS training course participants per 100,000 people ranged from 1,407 to 5,946 with the exception of one prefecture having a very high number. The proportion of aged population was $17.3-29.5 \%$. In correlation analysis, it was likely that the number of BLS training course participants per population negatively correlated to the survival rate $(-0.420$; -0.325 to -0.420$)$, and that the proportion of aged population weakly and positively correlated to the survival rate $(0.069$; 0.064-0.073).

The number of emergency hospitals per $100 \mathrm{~km}^{2}$ of residential area varied from 11.2 to 57.4. The number of ambulance teams per $100 \mathrm{~km}^{2}$ of residential area ranged from 21.0 to 63.4. The annual number of emergency medical dispatches per one ambulance ranged from 328 to 2,054. In correlation analysis, there was a very weak and negative correlation between each index and neurologically favourable 1-M survival: the number of emergency hospitals per $100 \mathrm{~km}^{2}$ of residential area $(-0.076 ;-0.081$ to -0.071$)$, number of ambulance teams per $100 \mathrm{~km}^{2}$ of residential area $(-0.169 ;-0.174$ to -0.164$)$, number of emergency medical dispatches per one ambulance $(-0.076$ to $-0.081)$.

The rates of tracheal intubation and adrenalin administration by paramedics widely varied: $0.7-25.5 \%$ and $4.1-27.7 \%$, respectively. In correlation analysis, there was positive correlation between each rate and neurologically favourable 1-M survival: tracheal intubation (0.528; 0.525-0.532), adrenalin administration (0.062; 0.057-0.067).

\section{Association of regional classifications by DA-CPR and EMS-related} indexes with neurologically favourable 1-month survival

Univariable analysis revealed that all regional classifications were significantly associated with neurologically favourable 1-M survival $(p<0.01$, Table 2$)$. We performed multinomial logistic regression where significant classifications from the univariable analysis were sequentially added to obtain the lowest BIC. The final best fit model included regional classifications based on sensitivity of DA-CPR, proportion of bystanders to follow DA-CPR, number of emergency hospitals, proportion of aged population, and numbers of emergency hospitals and ambulance teams per
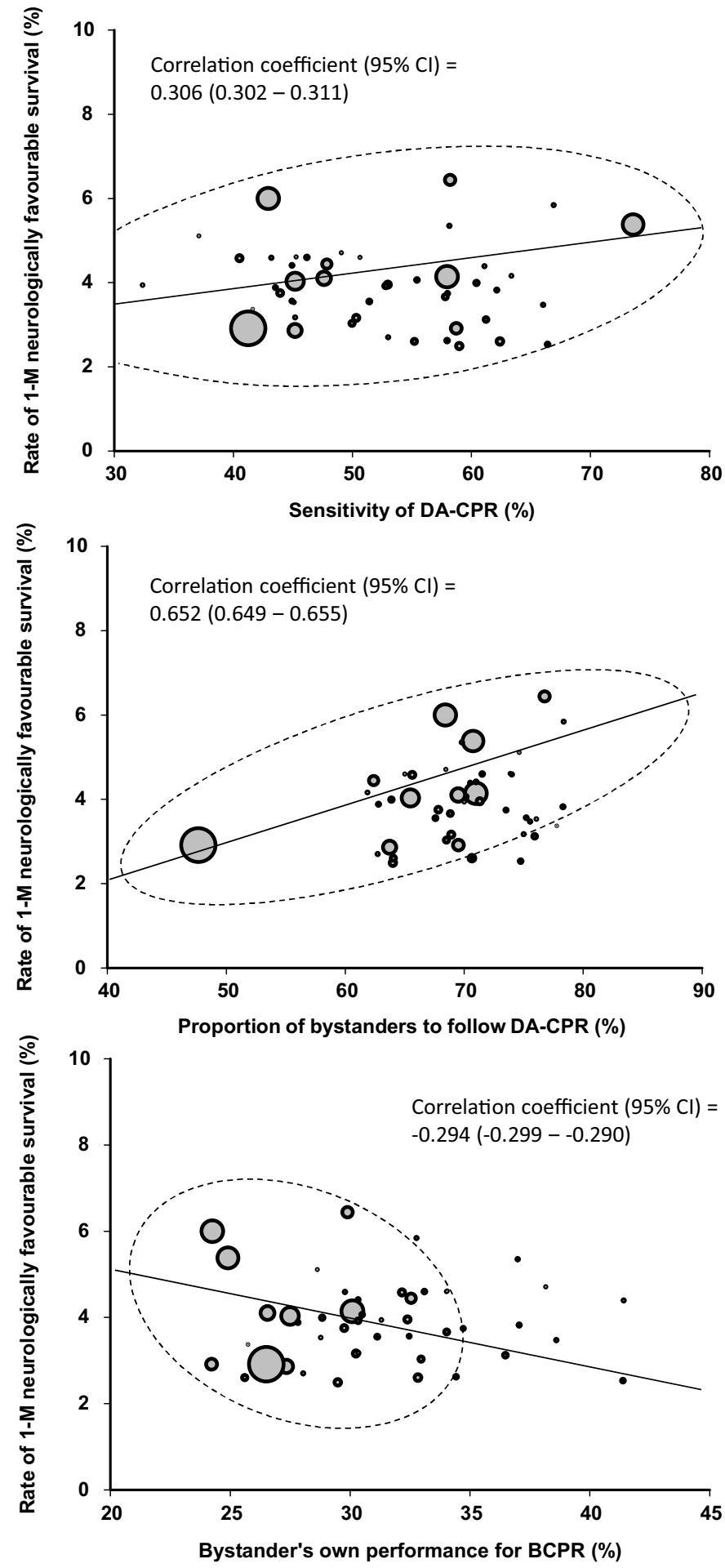

Fig. 2. Correlation between three DA-CPR- and BCPR-related indexes and OHCA survival. DA-CPR, dispatcher-assisted cardiopulmonary resuscitation; BCPR, bystander cardiopulmonary resuscitation. The size of each symbol reflects the number of outof-hospital cardiac arrests in each prefecture. Bivariate normal density ellipsoid $(\alpha=0.95)$ was presented.

$100 \mathrm{~km}^{2}$ of residential area. When the developing regions were set as reference, adjusted ORs $(95 \% \mathrm{CI})$ of the advanced region for sensitivity of DA-CPR and proportion of bystanders to follow DA-CPR were 1.615(1.410-1.851) and 1.713 (1.518-1.931), respectively. 
Table 2

Association of variation of BCPR- and EMS-related indexes with 1-M neurologically favourable survival.

\begin{tabular}{|c|c|c|c|c|}
\hline $\begin{array}{l}\text { Indexes/regions } \\
\text { (number of } \\
\text { prefectures) }\end{array}$ & $\begin{array}{l}\text { Regional value } \\
\text { of index } \\
\text { (range) }\end{array}$ & $\begin{array}{l}\text { 1-M } \\
\text { neurologically } \\
\text { favourable } \\
\text { survival rate }\end{array}$ & $\begin{array}{l}p \text { value/unadjusted } \\
\text { odds ratio }(95 \% \mathrm{CI}) \\
\text { for survival }\end{array}$ & $\begin{array}{l}\text { Adjusted odds ratio } \\
(95 \% \mathrm{CI}) \text { for survival }\end{array}$ \\
\hline $\begin{array}{l}\text { Sensitivity of DA-CPR, } \% \\
\text { Advanced prefectures }(N=5) \\
\text { Intermediate prefectures }(N=37) \\
\text { Developing prefectures }(N=5)\end{array}$ & $\begin{array}{l}70.9 \%(63.4-73.6) \\
51.7 \%(42.9-62.4) \\
40.6 \%(32.4-41.6)\end{array}$ & $\begin{array}{l}4.8 \%(781 / 16,232) \\
4.0 \%(4,737 / 118,422) \\
3.3 \%(734 / 22,439)\end{array}$ & $\begin{array}{l}p<0.001 / \\
1.495(1.349-1.657) \\
1.232(1.139-1.335) \\
\text { Reference }\end{array}$ & $\begin{array}{l}1.615(1.410-1.851) \\
1.399(1.254-1.561) \\
\text { Reference }\end{array}$ \\
\hline $\begin{array}{l}\text { Proportion of bystanders to follow DA-CPR, \% } \\
\text { Advanced prefectures }(N=5) \\
\text { Intermediate prefectures }(N=33) \\
\text { Developing prefectures }(N=9)\end{array}$ & $\begin{array}{l}77.3 \%(76.1-78.4) \\
70.0 \%(63.7-75.9) \\
57.7 \%(47.7-62.8)\end{array}$ & $\begin{array}{l}5.4 \%(527 / 9,697) \\
4.2 \%(4,967 / 106,319) \\
3.2 \%(758 / 41,077)\end{array}$ & $\begin{array}{l}p<0.001 / \\
1.761(1.587-1.953) \\
1.332(1.251-1.419) \\
\text { Reference }\end{array}$ & $\begin{array}{l}1.713(1.518-1.931) \\
1.233(1.142-1.332) \\
\text { Reference }\end{array}$ \\
\hline $\begin{array}{l}\text { Bystander's own performance of BCPR, \% } \\
\text { Advanced prefectures }(N=15) \\
\text { Intermediate prefectures }(N=26) \\
\text { Developing prefectures }(N=6)\end{array}$ & $\begin{array}{l}35.4 \%(32.8-41.4) \\
29.5 \%(26.6-32.8) \\
25.4 \%(24.2-26.5)\end{array}$ & $\begin{array}{l}3.5 \%(978 / 24,101) \\
4.0 \%(3,326 / 82,524) \\
4.2 \%(1,948 / 46,932)\end{array}$ & $\begin{array}{l}p<0.001 / \\
0.847(0.783-0.916) \\
0.970(0.916-1.027) \\
\text { Reference }\end{array}$ & Excluded \\
\hline $\begin{array}{l}\text { Number of BLS training course participants pe } \\
\text { Advanced prefectures }(N=10) \\
\text { Intermediate prefectures }(N=32) \\
\text { Developing prefectures }(N=5)\end{array}$ & $\begin{array}{l}100,000 \text { population } \\
3,039(2,356-5,946) \\
1,988(1,613-2,355) \\
1,515(1,407-1,585)\end{array}$ & $\begin{array}{l}3.9 \%(734 / 18,607) \\
3.9 \%(4,509 / 116,545) \\
4.6 \%(1,009 / 21,941)\end{array}$ & $\begin{array}{l}p<0.001 / \\
0.852(0.773-0.939) \\
0.835(0.779-0.896) \\
\text { Reference }\end{array}$ & Excluded \\
\hline $\begin{array}{l}\text { Proportion of aged ( } \geqq 65 \text { years) population, } \% \\
\text { Advanced prefectures }(N=11) \\
\text { Intermediate prefectures }(N=26) \\
\text { Developing prefectures }(N=10)\end{array}$ & $\begin{array}{l}27.3 \%(26.4-29.5) \\
24.2 \%(22.2-26.4) \\
20.7 \%(17.3-22.1)\end{array}$ & $\begin{array}{l}3.3 \%(593 / 18,018) \\
3.9 \%(2,410 / 63,549) \\
4.3 \%(3,249 / 75,526)\end{array}$ & $\begin{array}{l}p<0.001 / \\
0.757(0.692-0.827) \\
0.877(0.831-0.925) \\
\text { Reference }\end{array}$ & $\begin{array}{l}0.584(0.518-0.659) \\
0.720(0.653-0.794) \\
\text { Reference }\end{array}$ \\
\hline $\begin{array}{l}\text { Number of emergency hospitals per } 100 \mathrm{~km}^{2} \\
\text { Advanced prefectures }(N=11) \\
\text { Intermediate prefectures }(N=21) \\
\text { Developing prefectures }(N=15)\end{array}$ & $\begin{array}{l}\text { residential area } \\
43.7(32.4-57.4) \\
25.4(20.8-31.8) \\
16.7(11.2-19.9)\end{array}$ & $\begin{array}{l}4.2 \%(1,954 / 46,772) \\
4.4 \%(2,967 / 68,084) \\
3.2 \%(1,331 / 42,237)\end{array}$ & $\begin{array}{l}p<0.001 / \\
1.340(1.248-1.439) \\
1.400(1.311-1.496) \\
\text { Reference }\end{array}$ & $\begin{array}{l}1.260(1.143-1.389) \\
1.269(1.182-1.364) \\
\text { Reference }\end{array}$ \\
\hline $\begin{array}{l}\text { Number of ambulance teams per } 100 \mathrm{~km}^{2} \text { of re } \\
\text { Advanced prefectures }(N=5) \\
\text { Intermediate prefectures }(N=37) \\
\text { Developing prefectures }(N=5)\end{array}$ & $\begin{array}{l}\text { idential area } \\
58.0(55.2-63.4) \\
36.0(28.3-54.8) \\
24.5(21.0-27.5)\end{array}$ & $\begin{array}{l}4.2 \%(1,311 / 31,321) \\
4.0 \%(4,578 / 114,305) \\
3.2 \%(363 / 11,467)\end{array}$ & $\begin{array}{l}p<0.001 / \\
1.336(1.189-1.506) \\
1.276(1.147-1.425) \\
\text { Reference }\end{array}$ & $\begin{array}{l}1.481(1.257-1.746) \\
1.144(1.021-1.285) \\
\text { Reference }\end{array}$ \\
\hline $\begin{array}{l}\text { Annual number of emergency medical dispatc } \\
\text { Advanced prefectures }(N=5) \\
\text { Intermediate prefectures }(N=22) \\
\text { Developing prefectures }(N=20)\end{array}$ & $\begin{array}{l}\text { es per one ambulance } \\
1,563(1,141-2,054) \\
830(630-1,096) \\
523(328-628)\end{array}$ & $\begin{array}{l}4.6 \%(2,486 / 54,306) \\
3.6 \%(2,361 / 66,056) \\
3.8 \%(1,405 / 36,731)\end{array}$ & $\begin{array}{l}p<0.001 / \\
1.206(1.128-1.290) \\
0.932(0.871-0.997) \\
\text { Reference }\end{array}$ & Excluded \\
\hline $\begin{array}{l}\text { Rate of tracheal intubation by paramedics, \% } \\
\text { Advanced prefectures }(N=5) \\
\text { Intermediate prefectures }(N=37) \\
\text { Developing prefectures }(N=5)\end{array}$ & $\begin{array}{c}25.5 \%(22.2-26.4) \\
7.9 \%(3.0-18.3) \\
1.4 \%(0.7-1.6)\end{array}$ & $\begin{array}{l}5.2 \%(816 / 15,725) \\
3.7 \%(4,565 / 122,323) \\
4.6 \%(871 / 18,174)\end{array}$ & $\begin{array}{l}p<0.001 / \\
1.142(1.035-1.259) \\
0.809(0.752-0.872) \\
\text { Reference }\end{array}$ & Excluded \\
\hline $\begin{array}{l}\text { Rate of adrenaline administration by paramed } \\
\text { Advanced prefectures }(N=5) \\
\text { Intermediate prefectures }(N=37) \\
\text { Developing prefectures }(N=5)\end{array}$ & $\begin{array}{l}\text { s, \% } \\
27.7 \%(23.9-33.8) \\
14.1 \%(5.2-23.2) \\
4.1 \%(1.3-4.9)\end{array}$ & $\begin{array}{l}4.0 \%(615 / 15,578) \\
4.0 \%(5,378 / 134,089) \\
3.5 \%(259 / 7,426)\end{array}$ & $\begin{array}{l}p<0.001 / \\
1.137(0.982-1.321) \\
1.156(1.021-1.316) \\
\text { Reference }\end{array}$ & Excluded \\
\hline
\end{tabular}

DA-CPR, dispatcher-assisted cardiopulmonary resuscitation; BCPR, bystander cardiopulmonary resuscitation; 95\% CI, 95\% confidence interval Forty-seven prefectures were classified into three groups by ranking for each index: advanced, intermediate region, and developing regions.

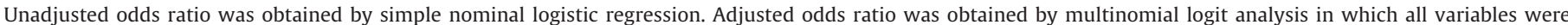
sequentially added to obtain the lowest Bayesian information criterion. Generalized $R^{2}$ of the final best-fit model was 0.0075 .

Patient factors associated with neurologically favourable 1-month survival

When univariable analysis was performed, age, gender, aetiology of arrest, performance of BCPR, initial rhythm, time interval between witnessing and emergency call, interval between emergency call and EMT arrival on site of OHCA, and interval between EMT arrival on site of OHCA and EMT arrival at hospital were associated with the survival (Table 3 ).

\section{Multivariable logistic regression analysis for neurologically} favourable 1-month survival (Fig. 3)

We confirmed the association between DA-CPR-related regional classifications and neurologically favourable 1-M survival by multivariable logistic regression analysis including both patient factors and regional classifications. Factors included in the final model were sensitivity of DA-CPR, proportion of bystanders to follow DA-CPR, provision of BCPR, patient age, aetiology of arrest, initial rhythm, the time interval between witness and emergency call, the time interval between emergency call and EMT arrival at patient, and number of emergency hospitals in residential area. Regarding sensitivity of DA-CPR, adjusted ORs $(95 \% \mathrm{CI})$ for survival with the developing region as reference was 1.277 (1.131-1.441) and 1.162 (1.058-1.277) for advanced and intermediate regions, respectively. Regarding the proportion of bystanders to follow DA-CPR, adjusted ORs for survival with the developing region as reference was 1.749 (1.554-1.967) and $1.280(1.188-1.297)$ for the advanced and intermediate regions, respectively.

We performed the same analyses in the two subgroups of OHCAs with and without tracheal intubation or adrenalin administration by paramedics (Supplementary Table). Both regional classifications by sensitivity of DA-CPR and proportion of bystanders to follow DACPR were associated with neurologically favourable $1-\mathrm{M}$ survival in 
Table 3

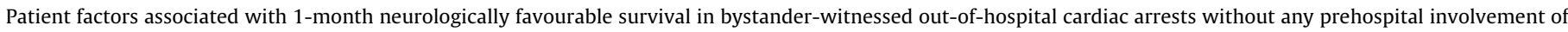
physician.

\begin{tabular}{|c|c|c|c|c|}
\hline & \multicolumn{2}{|c|}{$\begin{array}{l}\text { 1-month neurologically favourable } \\
\text { survival }\end{array}$} & \multirow{2}{*}{$\begin{array}{l}p \text { values by chi-square test } \\
\text { with Pearson's correction or } \\
\text { Wilcoxon rank sum test }\end{array}$} & \multirow{2}{*}{$\begin{array}{l}\text { Unadjusted odds ratio ( } 95 \% \\
\text { CI) for survival by multiple } \\
\text { logistic regression analysis }\end{array}$} \\
\hline & Yes $(N=6,252)$ & No $(N=150,841)$ & & \\
\hline Patient's age, year, median (IQR) & $64(52-74)$ & $78(66-86)$ & $p<0.0001$ & Undetermined \\
\hline $\begin{array}{l}\text { Patient's gender, } N(\%) \\
\text { Male } \\
\text { Female }\end{array}$ & $\begin{array}{l}4,676(74.8 \%) \\
1,576(25.2 \%)\end{array}$ & $\begin{array}{l}89,572(59.4 \%) \\
61,269(40.6 \%)\end{array}$ & $p<0.0001$ & $\begin{array}{l}2.029(1.915-2.151) \\
\text { Reference }\end{array}$ \\
\hline $\begin{array}{l}\text { Bystander CPR, } N(\%) \\
\text { Provided } \\
\text { Not provided }\end{array}$ & $\begin{array}{l}3,636(58.2 \%) \\
2,616(41.8 \%)\end{array}$ & $\begin{array}{l}68,659(45.5 \%) \\
82,182(54.5 \%)\end{array}$ & $p<0.0001$ & $\begin{array}{l}1.664(1.581-1.751) \\
\text { Reference }\end{array}$ \\
\hline $\begin{array}{l}\text { Aetiology of arrest, } N(\%) \\
\text { Presumed cardiac } \\
\text { Presumed noncardiac }\end{array}$ & $\begin{array}{l}5,012(80.2 \%) \\
1,240(19.8 \%)\end{array}$ & $\begin{array}{l}82,276(54.5 \%) \\
68,565(45.5 \%)\end{array}$ & $p<0.0001$ & $\begin{array}{l}3.368(3.163-3.587) \\
\text { Reference }\end{array}$ \\
\hline $\begin{array}{l}\text { Initial rhythm, } N(\%) \\
\text { Shockable } \\
\text { Non-shockable }\end{array}$ & $\begin{array}{l}3,967(63.5 \%) \\
2,285(36.6 \%)\end{array}$ & $\begin{array}{r}17,441(11.6 \%) \\
133,400(88.4 \%)\end{array}$ & $p<0.0001$ & $\begin{array}{l}13.28(12.58-14.01) \\
\text { Reference }\end{array}$ \\
\hline $\begin{array}{l}\text { Time intervals, min, median (IQR) } \\
\text { Witness-call } \\
\text { Call-EMT arrival at patient } \\
\text { EMT arrival at patient-EMT arrival at hospital }\end{array}$ & $\begin{array}{l}1(0-3) \\
7(5-9) \\
20(16-27)\end{array}$ & $\begin{array}{c}2(0-5) \\
8(6-10) \\
22(17-29)\end{array}$ & $\begin{array}{l}p<0.0001 \\
p<0.0001 \\
p<0.0001\end{array}$ & $\begin{array}{l}\text { Undetermined } \\
\text { Undetermined } \\
\text { Undetermined }\end{array}$ \\
\hline
\end{tabular}

IQR, interquartile range; BCPR, bystander cardiopulmonary resuscitation; 95\% CI, 95\% confidence interval, EMT; emergency medical technician.

Unadjusted odds ratio was obtained by simple nominal logit analysis.

the subgroup without advanced life support. Only the classification by sensitivity of DA-CPR was associated with the survival in the subgroup with advanced life support.

\section{Discussion}

The present study showed that regional variations in sensitivity of DA-CPR and the proportion of bystanders to follow DA-CPR were associated with neurologically favourable 1-M survival after bystander-witnessed OHCAs without any prehospital physician involvement. Surprisingly, regional classification by bystander's performance of BCPR without instruction or number of BLS training course participants per population was not associated with survival.

In Japan, fire departments play a major role in educating citizens regarding BLS. Every year, approximately 1.5 million citizens participate BLS training courses conducted by fire departments. ${ }^{13}$ Furthermore, approximately 1.1 million new driver license applicants are required to undergo BLS training in authorized driving schools. ${ }^{15}$ Supposing that the participants in these training courses may maintain sufficient skill and willingness to perform BCPR for three years, $5.7 \%$ of population are expected to perform BCPR if the need arises. The total number of healthcare providers and care facility staff were estimated to be 3.5 million in $2011 .^{20}$ Thus, the proportion of potential BLS providers in the population will be less than $10 \%$ of population even when the estimation is the highest. Furthermore, not all BLS-trained bystanders perform CPR by their own initiative. ${ }^{21}$ Therefore, as in other countries, ${ }^{8,21-23}$ performance of DA-CPR is one of the major determinants of BCPR in Japan. Indeed, only $36 \%(25,938 / 72,295)$ of BCPRs were initiated without DA-CPR in this study.

In Japan, DA-CPR has been introduced since the release of DACPR guidelines by FDMA in $1999 .{ }^{24}$ Medical control system for EMS was implemented in Japan when trained paramedics were allowed to perform tracheal intubation in $2004 .{ }^{25}$ It was very recent (in 2013) that the Japanese DA-CPR guidelines including the standardized protocol and educational approach to the dispatchers' training were released from FDMA. ${ }^{24}$ Therefore, quality improvement and training programmes for DA-CPR ${ }^{26}$ was introduced under medical control only in some prefectures. Education and qualification of dispatchers, which are established in other countries, ${ }^{27,28}$ have not been implemented in Japan. The sensitivity of DA-CPR is known to be influenced by dispatcher's perceptions of providing DA-CPR and the trustworthiness of the witnesses ${ }^{8,29}$ that may be affected by regional differences in backgrounds of callers and resources of the dispatch centre involved in DA-CPR. Therefore, further nation-wide educational approach to dispatchers and quality improvement programme for DA-CPR may be needed to correct the regional variations in Japan.

Regional variation in the proportion of bystanders to follow DACPR was smaller than that in sensitivity of DA-CPR. These rates in Japan are much higher than those reported from three cities in the United States ${ }^{30}$ and comparable with those reported from other cities. $^{31,32}$ Although various reasons for callers not providing BCPR have been reported, ${ }^{30,32}$ the proportion of bystanders to follow DACPR is also dependent on the skill of dispatchers to provide a quick just-in-time instruction ${ }^{33}$ encouraging the callers.

Considerable regional variations in bystander's performance of CPR without instruction and number of BLS training course participants were observed. However, regional variation in quantity of bystander's performance of BCPR or in number of participants in BLS training courses was not associated with neurologically favourable 1-M survival in this study. It is controversial whether BCPR training alone may improve the survival after OHCAs. Emphasis on widespread CPR teaching in rural areas may not be justifiable. ${ }^{34}$ As reported in Denmark, ${ }^{35}$ nationwide educational efforts towards both citizens and dispatchers would improve the outcomes of OHCAs.

This study has several limitations. The data were analysed in terms of prefectures. Similar regional variations may exist internally in each prefecture. The FDMA database had no information on dispatcher performance or reasons for no DA-CPR provision and refusal of DA-CPR. No data on BCPR quality, known to be a major factor in achieving better OHCA outcomes, ${ }^{2}$ were collected. Therefore, unmeasured factors associated with DA-CPR and BCPR may influence the results. The final outcomes were measured at one month, although a longer observation period has been recommended. ${ }^{36}$ However, this study contains a notably large prospective cohort, 


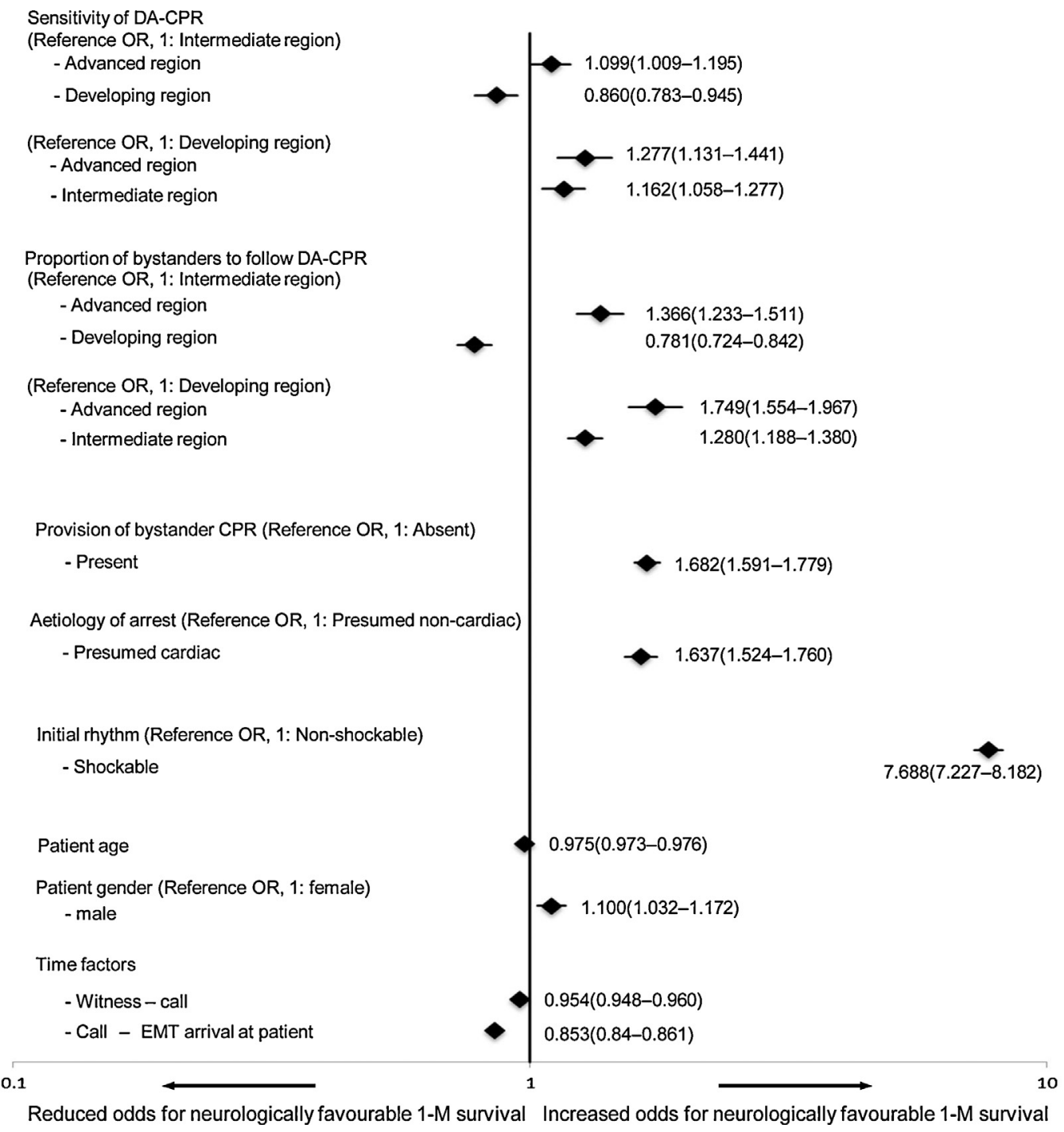

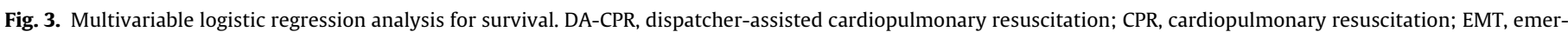

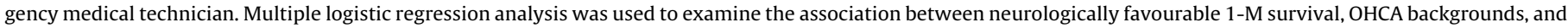

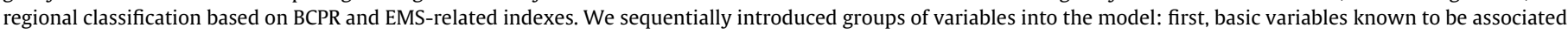

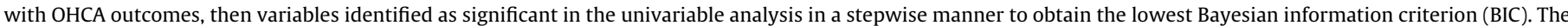
generalized $R^{2}$ of the final model was 0.2559 .

and the results of this study suggest the requirement of a national initiative to establish a better DA-CPR protocol with qualified educational system for dispatchers and to improve the compliance with DA-CPR protocol. ${ }^{37}$

\section{Conclusion}

Wide variations in sensitivity of DA-CPR and proportion of bystanders to follow DA-CPR exist in Japan. Good outcomes of bystander-witnessed OHCAs correlate with regions providing DACPR with higher sensitivity of DA-CPR and higher proportion of bystanders to follow DA-CPR. Nation-wide educational approach to dispatchers and quality improvement programme for DA-CPR may be needed to correct the regional variations in Japan.

\section{Conflict of interest}

The authors declare no conflicts of interest associated with this manuscript.

\section{Acknowledgements}

We would like to thank all of the EMS personnel and the FDMA in Japan for their great cooperation in collecting and managing the Utstein-style database.

\section{Appendix A. Supplementary data}

Supplementary data associated with this article can be found, in the online version, at http://dx.doi.org/10.1016/j.resuscitation. 2015.10.004.

\section{References}

1. Wang HE, Devlin SM, Sears GK, et al. Regional variations in early and late survival after out-of-hospital cardiac arrest. Resuscitation 2012;83:1343-8.

2. Nichol G, Thomas E, Callaway CW, et al. Regional variation in out-of-hospital cardiac arrest incidence and outcome. JAMA 2008;300:1423-31.

3. Berdowski J, Berg RA, Tijssen JG, Koster RW. Global incidences of out-of-hospital cardiac arrest and survival rates: systematic review of 67 prospective studies. Resuscitation 2010;81:1479-87. 
4. Carr BG, Kahn JM, Merchant RM, Kramer AA, Neumar RW. Inter-hospital variability in post-cardiac arrest mortality. Resuscitation 2009;80:30-4.

5. Stiell I, Nichol G, Wells G, et al. Health-related quality of life is better for cardiac arrest survivors who received citizen cardiopulmonary resuscitation. Circulation 2003;108:1939-44

6. Eisenberg MS, Bergner L, Hallstrom A. Cardiac resuscitation in the community. Importance of rapid provision and implications for program planning. JAMA 1979;241:1905-7.

7. Tanaka Y, Taniguchi J, Wato Y, Yoshida Y, Inaba H. The continuous quality improvement project for telephone-assisted instruction of cardiopulmonary resuscitation increased the incidence of bystander CPR and improved the outcomes of out-of-hospital cardiac arrests. Resuscitation 2012;83:1235-41.

8. Kuisma M, Boyd J, Väyrynen T, Repo J, Nousila-Wiik M, Holmström P. Emergency call processing and survival from out-of-hospital ventricular fibrillation. Resuscitation 2005;67:89-93.

9. Takei Y, Nishi T, Matsubara H, Hashimoto M, Inaba H. Factors associated with quality of bystander CPR: the presence of multiple rescuers and bystanderinitiated CPR without instruction. Resuscitation 2014;85:492-8.

10. Tanaka Y, Nishi T, Takase K, et al. Survey of a protocol to increase appropriate implementation of dispatcher-assisted cardiopulmonary resuscitation for outof-hospital cardiac arrest. Circulation 2014;129:1751-60.

11. Jacobs I, Nadkarni V, Bahr J, et al. Cardiac arrest and cardiopulmonary resuscitation outcome reports: update and simplification of the Utstein templates for resuscitation registries: a statement for healthcare professionals from a task force of the International Liaison Committee on Resuscitation (American Heart Association, European Resuscitation Council, Australian Resuscitation Council, New Zealand Resuscitation Council, Heart and Stroke Foundation of Canada, Inter American Heart Foundation, Resuscitation Councils of Southern Africa). Circulation 2004;110:3385-97.

12. Ministry of Internal Affairs and Communications Statics Bureau, Directorgeneral for Policy Planning (Statistical Standards) \& Statistical Research and Training Institute. http://www.stat.go.jp/english/data/jinsui/tsuki/index.htm; 2014 [accessed 25.05.14].

13. Fire and Disaster Management Agency. http://www.fdma.go.jp/en/; 2014 [accessed 25.05.14].

14. Enami M, Takei Y, Inaba H, et al. Differential effects of ageing and BLS training experience on attitude towards basic life support. Resuscitation 2011; 82:577-83.

15. Driver's License Statistics. http://www.npa.go.jp/toukei/menkyo/menkyo13/ h22_main.pdf; 2014 [accessed 25.05.14].

16. Japan Resuscitation Council, JRC Guidelines, 2010. Available from: http://jrc. umin.ac.jp/english.html [accessed 25.05.14].

17. Yasunaga $\mathrm{H}$, Horiguchi $\mathrm{H}$, Tanabe $\mathrm{S}$, et al. Collaborative effects of bystanderinitiated cardiopulmonary resuscitation and prehospital advanced cardiac life support by physicians on survival of out-of-hospital cardiac arrest: a nationwide population-based observational study. Crit Care 2010;14:R199.

18. Jennett B, Bond M. Assessment of outcome after severe brain damage. Lancet $1975 ; 1: 480-4$

19. Preacher, K.J. Calculation for the chi-square test: An interactive calculation tool for chi-square tests of goodness of fit and independence [Computer software]. Available from: http://quantpsy.org./chisq/chisq; 2001.
20. Ministry of Health, Labour and Welfare. http://www.mhlw.go.jp/file.jsp?id= 146268\&name=2r98520000033t98_1.pdf; 2014 [accessed 20.08.14].

21. Swor R, Khan L, Domeier R, Honeycutt L, Chu K, Compton S. CPR training and CPR performance: do CPR-trained bystanders perform CPR? Acad Emerg Med 2006;13:596-601.

22. Berdowski J, Beekhuis F, Zwinderman AH, Tijssen JG, Koster RW. Importance of the first link: description and recognition of an out-of-hospital cardiac arrest in an emergency call. Circulation 2009;119:2096-102.

23. Bohm K, Vaillancourt C, Charette ML, Dunford J, Castrén M. In patients with out-of-hospital cardiac arrest, does the provision of dispatch cardiopulmonary resuscitation instructions as opposed to no instructions improve outcome: a systematic review of the literature. Resuscitation 2011;82:1490-5.

24. Fire and Disaster Management Agency. http://www.fdma.go.jp/emergency rescue/kyukyu_kyujo_tuchi/2013/20130517-10.pdf; 2014 [accessed 20.08.14].

25. Fire and Disaster Management Agency. http://www.fdma.go.jp/html/data/ tuchi1407/140723syou160.htm; 2014 [accessed 20.08.14].

26. Calle PA, Lagaert L, Vanhaute O, Buylaert WA. Do victims of an out-of-hospital cardiac arrest benefit from a training program for emergency medical dispatchers? Resuscitation 1997;35:213-8.

27. Bureau of Labor Statistics, U.S. Department of Labor, Occupational Outlook Handbook, 2012-13 Edition, Police, Fire, and Ambulance Dispatchers. http://www.bls.gov/ooh/office-and-administrative-support/police-fire-andambulance-dispatchers.htm; [accessed 20.08.14].

28. Yancey A, Clawson J. EMD position paper resource document. AEDR 2014;2: 33-40.

29. Bang A, Ortgren P-O, Herlitz J, Währborg P. Dispatcher-assisted telephone CPR: a qualitative study exploring how dispatchers perceive their experiences. Resuscitation 2002;53:135-51.

30. Lerner EB, Sayre MR, Brice JH, et al. Cardiac arrest patients rarely receive chest compressions before ambulance arrival despite the availability of pre-arrival CPR instructions. Resuscitation 2008;77:51-6.

31. Eisenberg MS, Hallstrom AP, Carter WB, Cummins RO, Bergner L, Pierce J. Emergency CPR instruction via telephone. Am J Public Health 1985;75:47-50.

32. O'Neill JF, Deakin CD. Evaluation of telephone CPR advice for adult cardiac arres patients. Resuscitation 2007;74:63-7.

33. Lerner EB, Rea TD, Bobrow BJ, et al. Emergency medical service dispatch cardiopulmonary resuscitation prearrival instructions to improve survival from out-of-hospital cardiac arrest: a scientific statement from the American Heart Association. Circulation 2012:125:648-55.

34. Jennings PA, Cameron P, Walker T, Bernard S, Smith K. Out-of-hospital cardiac arrest in Victoria: rural and urban outcomes. Med J Aust 2006;185:135-9.

35. Wissenberg M, Lippert FK, Folke F, et al. Association of national initiatives to improve cardiac arrest management with rates of bystander intervention and patient survival after out-of-hospital cardiac arrest. JAMA 2013;310: $1377-84$.

36. Arrich J, Zeiner A, Sterz F, et al. Factors associated with a change in functiona outcome between one month and six months after cardiac arrest: a retrospective cohort study. Resuscitation 2009;80:876-80.

37. Clawson JJ, Cady GA, Martin RL, et al. Effect of a comprehensive quality management process on compliance with protocol in an emergency medical dispatch center. Ann Emerg Med 1998;32:578-84. 